\title{
Modeling and Analytical Simulation of a Smouldering Carbonaceous Rod
}

\section{*11MOHAMMED, AA; ${ }^{2}$ OLAYIWOLA, RO; ${ }^{3}$ ESEYIN, M; ${ }^{4}$ WACHIN, AA}

\author{
${ }^{123}$ Department of Mathematics, Federal University of Technology, Minna, Nigeria. \\ ${ }^{4}$ Department of Mathematics/Computer Science, Ibrahim Badamasi Babangida Unversity, Lapai, \\ Nigeria. \\ E-mail.: adamu.alhaj@futminna.edu.ng,olayiwolarasaq@yahoo.co.uk, aawachin@gmail.com
}

\begin{abstract}
Modeling of pyrolysis and combustion in a smouldering fuel bed requires the solution of flow, heat and mass transfer through porous media. This paper presents an analytical method for describing the smouldering of a porous carbonaceous rod. We assume that no local thermal equilibrium exist between the phases. Also, the initial temperatures are assumed to depend on space variable. We prove the existence and uniqueness of solution of the model by actual solution method. The coupled non-linear partial differential equations describing the phenomenon have been decoupled using parameter-expanding method and solved analytically using eigenfunction expansion technique. The results obtained were discussed. The study shows that the Frank-Kamenetskii number, Scaled thermal conductivity of gas and solid phases and Species diffusion coefficient have a significant effects on the slow burning process associated with porous carbonaceous rod. (C) JASEM
\end{abstract}

https://dx.doi.org/10.4314/jasem.v21i5.21

Keywords: Pyrolysis, smouldering, combustion, carbonaceous rod, pyrolysis reactions, analytical solution.

Smouldering is the slow, low-temperature, flameless form of combustion of a condensed fuel. It poses safety and environmental hazards and allows novel technological application but its fundamentals remain mostly unknown to the scientific community (Rein, 2009). The terms filtering combustion, smoking problem, deep seated fires, hidden fires, peat or peat lands fires, lagging fires, low oxygen combustion, insitu combustion, fireflood and underground gasification, all refer to smouldering combustion phenomena (Rein, 2009). Smouldering is the leading cause of deaths in residential fires (Rein, 2009; Hall, 2004) and a source of safety concerns in space and commercial flights (Rein, 2009). It is of interest both as a fundamental combustion problem and as a practical fire hazard (Hall, 2004). Smouldering wildfires destroy large amounts of biomass and cause great damage to the soil, contributing significantly to atmospheric pollutant and greenhouse gas emissions (Rein, 2009).

Smouldering of a porous carbonaceous rod is normally controlled by two main parameters availability of oxygen to the combustion front and the heat losses from it. The velocity of the combustion front into the carbonaceous fuel after the ignition by an external heat source and the peak temperature are two indicators of the sustenance of combustion. It is a transient process which is controlled by a combination of endothermic and exothermic chemical reactions in the pyrolysis and combustion zones, diffusion of oxygen to the combustion zone, diffusion of reaction products away from the sources, and heat transfer as well (Rostami et al., 2002).
Several works have been done on smouldering combustion. These include the work of Ohlemiller (1985) who considered a burning cigarette as a forward smouldering case, in which the air flow is in the same direction as the travelling combustion front. Kinbara et al. (1967) used a diffusion controlled approach to model the smouldering of a cylindrical fuel. Olayiwola (2012) studied transient onedimensional governing equations for smouldering combustion in a porous fuel. He assumed that there is a perfect contact between the gas and solid phases. He considered the pressure gradient to be parabolic and proved the existence and uniqueness of solution of the model by actual solution. In another development, Olayiwola et al. (2013) considered steady one-dimensional governing equations for smouldering combustion in a porous fuel. $\mathrm{He}$ examined the properties of solution of the steadystate problems under certain conditions and investigated the effect of Frank-Kamenetskii parameter on the propagation of forward and opposed smouldering combustion.

The objective of this paper is to obtain an analytical solution for describing the smouldering of a porous carbonaceous rod.

\section{MATERIALS AND METHODS}

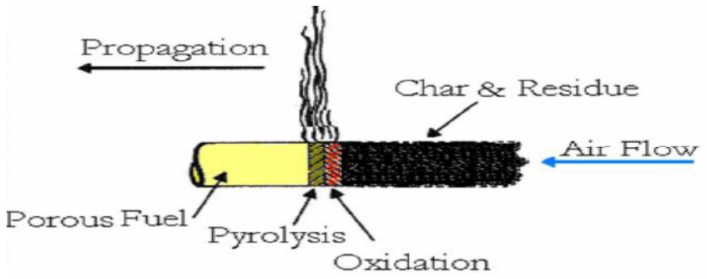

Fig 1: One-dimensional reaction front in forward smoldering and the correspondence in a burning cigarette (Rein, 2009). 
We consider a porous carbonaceous rod as shown in fig 1. The gas and solid phases are treated separately. Pyrolysis occurs in the starting material through a set of pyrolysis reactions. The pyrolysis reactions result in the formation of a series of products. The pyrolysis products leave the solid phase, while the remaining carbonaceous residue is oxidized when exposed to high temperatures and oxygen. The remaining solid residues that react with oxygen at high temperature are assumed to follow Arrhenius relation. We assume that the initial temperatures depend on space variable $x$. With the above assumptions, the transient, onedimensional governing equations for the natural smouldering of a porous carbonaceous rod are:

Conservation of energy of solid:

$$
\begin{aligned}
& (1-\phi) \frac{\partial}{\partial t}\left(\rho_{s} c_{p s} T_{s}\right)=\frac{\partial}{\partial x}\left(k_{s}(1-\phi) \frac{\partial T_{s}}{\partial x}\right) \\
& +\frac{h_{g s} A_{g s}}{V}\left(T_{g}-T_{s}\right)-\rho_{0} \Delta h A e^{-\frac{E}{R T_{s}}}
\end{aligned}
$$

\section{Conservation of energy of gas:}

$$
\begin{aligned}
& \frac{\partial}{\partial t}\left(\phi \rho_{g} c_{p g} T_{g}\right)=\frac{\partial}{\partial x}\left(\phi k_{g} \frac{\partial T_{g}}{\partial x}\right)+ \\
& \frac{\partial}{\partial x}\left(\phi \rho_{g} c_{p g}\left(T_{g}-T_{0}\right) \frac{K}{\mu} \frac{\partial P}{\partial x}\right)- \\
& \frac{h_{g s} A_{g s}}{V}\left(T_{g}-T_{s}\right)
\end{aligned}
$$

\section{Conservation of gas species (Oxygen):}

$\frac{\partial}{\partial t}\left(\phi \rho_{g} Y_{o x}\right)=\frac{\partial}{\partial x}\left(\phi \rho_{g} D \frac{\partial Y_{o x}}{\partial x}\right)+$

$\frac{\partial}{\partial x}\left(\phi \rho_{g} \frac{K}{\mu} \frac{\partial P}{\partial x} Y_{o x}\right)-\rho_{0} v_{o x} A e^{-\frac{E}{R T s}}$

Smouldering product:

$$
\begin{aligned}
& \frac{\partial}{\partial t}\left(\phi \rho_{g} Y_{g p}\right)=\frac{\partial}{\partial x}\left(\phi \rho_{g} D \frac{\partial Y_{g p}}{\partial x}\right)+ \\
& \frac{\partial}{\partial x}\left(\phi \rho_{g} \frac{K_{x}}{\mu} \frac{\partial P}{\partial x} Y_{g p}\right)+\rho_{0} v_{g p} A e^{-\frac{E}{R T_{s}}}
\end{aligned}
$$

The initial and boundary conditions were formulated as follows: Initial condition:

At $t=0$ and $\forall x$

$\left.\begin{array}{c}T_{s}=\frac{R T_{0}^{2}}{E}\left(1-\frac{x}{L}\right)+T_{0}, T_{g}=\frac{R T_{0}^{2}}{E}\left(1-\frac{x}{L}\right)+T_{0} \\ Y_{g p}=0\end{array}\right\}$

Boundary conditions:

$$
\left.\begin{array}{ll}
\left.T_{s}\right|_{x=0}=T_{0}, & \left.T_{s}\right|_{x=L}=T_{0} \\
\left.T_{g}\right|_{x=0}=T_{0}, & \left.T_{g}\right|_{x=L}=T_{0}
\end{array}\right\}
$$

where $\phi$ is the porosity of the porous bed, $\frac{A_{g s}}{V}$ is the ratio of surface area between gas and solid to volume, $E$ is activation energy, $R$ is the perfect gas constant, $L$ is the length of porous bed, $k$ is thermal conductivity, $\Delta h$ is the enthalpy of reaction, $c$ is specific heat, $T$ is temperature, $Y$ is the mass fraction of the species, $t$ is time, $x$ is position, $h_{g s}$ is the heat transfer coefficient between gas and solid, $D$ is the diffusion coefficient of the species, $K$ is permeability, $\mu$ is dynamic viscosity, $P$ is pressure, $\rho$ is density, $A$ is the pre-exponential factor and the subscripts $s, g, p, o x, g p$ and 0 represent solid, gas, pressure, oxygen, gas product and initial respectively.

\section{Method of Solution}

In our analysis, we consider the pressure gradient to be parabolic (Olayiwola, 2012; Olayiwola et al., 2013), i.e.:

$$
\begin{aligned}
& \frac{\partial P}{\partial x}=f(x)=\frac{x}{L}\left(1-\frac{x}{L}\right) \\
& \text { and } \quad \text { assume } \quad \rho_{s}, \rho_{g}, \rho_{0}, c_{p}, c_{p g}, c_{p s}, \\
& k_{s}, k_{g}, \phi, D, K, K_{x} \text { and } \mu \text { to be constants. }
\end{aligned}
$$

These assumptions could be relaxed in the future.

\section{Non-dimensionalization}

Here, we non-dimensionalized equations (1) - (7), using the following dimensionless variables:

$$
\left.\begin{array}{c}
x^{\prime}=\frac{x}{L}, t^{\prime}=\frac{t}{t_{0}}, Y=\frac{Y_{o x}}{Y_{0}}, Z=\frac{Y_{g p}}{Y_{0}}, \epsilon=\frac{R T_{0}}{E}, \\
\theta=\frac{E}{R T_{0}^{2}}\left(T_{s}-T_{0}\right), \quad \varphi=\frac{E}{R T_{0}^{2}}\left(T_{g}-T_{0}\right)
\end{array}\right\}
$$

where $t_{0}$ is the initial time for ignition to occur and we obtain

$$
\begin{aligned}
\frac{\partial \theta}{\partial t}= & \lambda_{1} \frac{\partial^{2} \theta}{\partial x^{2}}+\alpha_{1}(\varphi-\theta)- \\
& \delta \exp \left(\frac{\theta}{1+\epsilon \theta}\right) \\
\frac{\partial \varphi}{\partial t}= & \lambda_{2} \frac{\partial^{2} \varphi}{\partial x^{2}}+k_{1} x(1-x) \frac{\partial \varphi}{\partial x}+ \\
& k_{1}(1-2 x) \varphi-\alpha_{2}(\varphi-\theta) \\
\frac{\partial Y}{\partial t}= & D_{1} \frac{\partial^{2} Y}{\partial x^{2}}+k_{2} x(1-x) \frac{\partial Y}{\partial x}+ \\
& k_{2}(1-2 x) Y-\beta \exp \left(\frac{\theta}{1+\epsilon \theta}\right) \\
\frac{\partial Z}{\partial t}= & D_{1} \frac{\partial^{2} Z}{\partial x^{2}}+k_{2} x(1-x) \frac{\partial Z}{\partial x}+ \\
& k_{2}(1-2 x) Z+\sigma \exp \left(\frac{\theta}{1+\epsilon \theta}\right)
\end{aligned}
$$

Together with initial and boundary conditions: $\theta(x, 0)=1-x, \theta(0, t)=0, \quad \theta(1, t)=0$ $\varphi(x, 0)=1-x, \varphi(0, t)=0, \varphi(1, t)=0$ $Y(x, 0)=0, \quad Y(0, t)=1, \quad Y(1, t)=0$ $Z(x, 0)=0, \quad Z(0, t)=0, \quad Z(1, t)=0$

where 
$\lambda_{1}=\frac{k_{s} t_{0}}{L^{2} \rho_{s} c_{p s}}=$ Solid phase scaled thermal

conductivity, $\lambda_{2}=\frac{k_{g} t_{0}}{L^{2} \rho_{g} c_{p g}}=$ Gas phase scaled thermal conductivity, $\quad \alpha_{1}=\frac{h_{g s} A_{g s} t_{0}}{(1-\phi) \rho_{s} c_{p s} V}$, $\alpha_{2}=\frac{h_{g s} A_{g s} t_{0}}{\phi \rho_{g} c_{p g} V}, \quad \delta=\frac{\rho_{0} \Delta h A t_{0} e^{-\frac{E}{R T}}}{(1-\phi) \rho_{s} c_{p s} \in T_{0}}=$ Frank-Kamenetskii parameter, $\quad k_{1}=\frac{K t_{0}}{L \mu}$, $k_{2}=\frac{K_{x} t_{0}}{L \mu}, \quad D_{1}=\frac{D t_{0}}{L^{2}}=$ Species diffusion coefficient,

$$
\beta=\frac{\rho_{0} v_{o x} A t_{0} e^{-\frac{E}{R T_{0}}}}{\phi \rho_{g} Y_{0}},
$$

$\sigma=\frac{\rho_{0} v_{g p} A t_{0} e^{-\frac{E}{R T_{0}}}}{\phi \rho_{g} Y_{0}}$

\section{Analytical Solution}

We solve equations (10) - (14) using parameterexpanding method (where details can be found in (He, 2006)) and eigenfunctions expansion method (where details can be found in (Myint-U and Debnath, 1987)).

Using an approximation of the form (Ayeni (1982)):

$\exp \left(\frac{\theta}{1+\in \theta}\right) \approx 1+(e-2) \theta$

and parameter expanding method and eigenfunctions expansion technique, we obtain the solutions of equations (10) - (14) as

$\theta_{0}(x, t)=\sum_{n=1}^{\infty}\left(A+(B-A) e^{-C t}\right) \sin n \pi x$

$\varphi_{0}(x, t)=\sum_{n=1}^{\infty}\left(\begin{array}{l}B e^{-C_{1} t}+ \\ \left.\alpha_{2} \sum_{n=1}^{\infty}\left(\begin{array}{c}B_{1}\left(1-e^{-C_{1} t}\right) \\ +B_{2}\left(\begin{array}{l}e^{-C t}- \\ e^{-C_{1} t}\end{array}\right)\end{array}\right)\right) \sin n \pi x\end{array}\right.$

$Y_{0}(x, t)=(1-x)+$

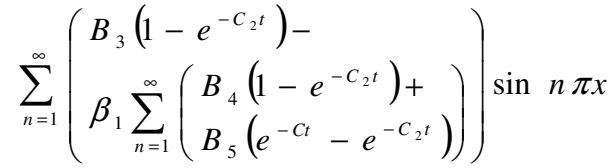

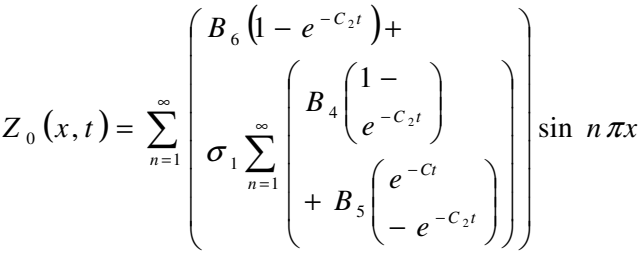

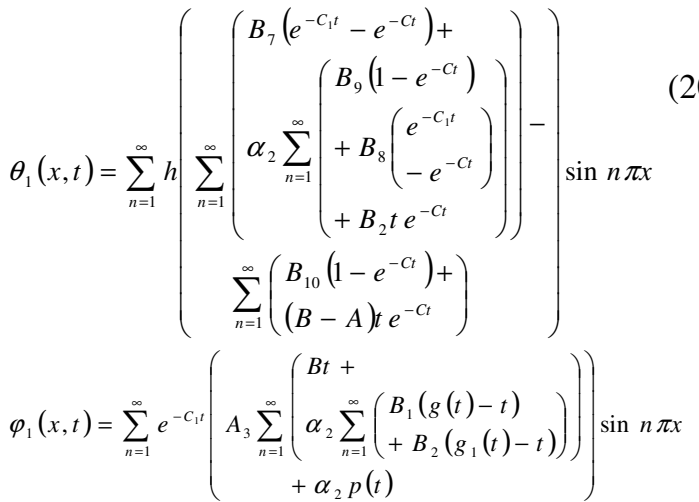

(21)

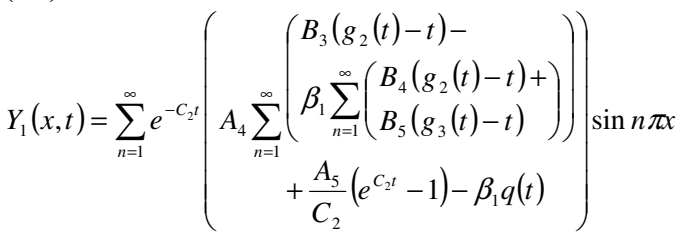

$Z_{1}(x, t)=\sum_{n=1}^{\infty} e^{-C_{2} t}\left(A_{4} \sum_{n=1}^{\infty}\left(\begin{array}{c}B_{6}\left(g_{2}(t)-t\right)+ \\ \left.\sigma_{1} \sum_{n=1}^{\infty}\left(\begin{array}{l}B_{4}\left(g_{2}(t)-t\right) \\ +B_{5}\left(g_{3}(t)-t\right)\end{array}\right)\right)+\sin n \pi x \\ \sigma_{1} q(t)\end{array}\right)\right.$

where

$p(t)=\sum_{n=1}^{\infty} h\left(\sum_{n=1}^{\infty}\left(\begin{array}{c}\alpha_{2} \sum_{n=1}^{\infty}\left(\begin{array}{l}B_{7}\left(t-g_{1}(t)\right)+ \\ B_{8}\left(t-g_{1}(t)\right)+ \\ \frac{B_{2}}{C_{3}}\left(t e^{-C_{3} t}-g_{1}(t)\right)\end{array}\right) \\ \sum_{n=1}^{\infty}\left(\begin{array}{l}B_{10}\left(g(t)-g_{1}(t)\right)+ \\ \frac{(B-A)}{C_{3}}\left(t e^{-C_{3} t}-g_{1}(t)\right)\end{array}\right)\end{array}\right)-\right.$

$$
q(t)=\sum_{n=1}^{\infty} h\left(\sum_{n=1}^{\infty}\left(\begin{array}{c}
B_{7}\left(g_{4}(t)-g_{3}(t)\right)+ \\
\alpha_{2} \sum_{n=1}^{\infty}\left(\begin{array}{l}
B_{9}\left(g_{2}(t)-g_{3}(t)\right) \\
B_{8}\left(g_{4}(t)-g_{3}(t)\right) \\
+\frac{B_{2}}{C_{4}}\left(t e^{-C_{4} t}-g_{3}(t)\right)
\end{array}\right) \\
\sum_{n=1}^{\infty}\left(\begin{array}{l}
B_{10}\left(g_{2}(t)-g_{3}(t)\right)+ \\
\frac{(B-A)}{C_{4}}\left(t e^{-C_{4} t}-g_{3}(t)\right)
\end{array}\right)
\end{array}\right)-\right.
$$




$$
\begin{aligned}
& g(t)=\frac{1}{C_{1}}\left(e^{C_{1} t}-1\right), \quad g_{1}(t)=\frac{1}{C_{3}}\left(e^{C_{3} t}-1\right), \\
& g_{2}(t)=\frac{1}{C_{2}}\left(e^{C_{2} t}-1\right), g_{3}(t)=\frac{1}{C_{4}}\left(e^{C_{4} t}-1\right) \text {, } \\
& g_{4}(t)=\frac{1}{C_{5}}\left(e^{C_{5} t}-1\right), C_{3}=C_{1}-C, \\
& C_{4}=C_{2}-C, \quad C_{5}=C_{2}-C_{1}, \\
& \alpha=\delta(e-2), \quad A=\frac{2 \delta\left((-1)^{n}-1\right)}{n \pi C}, \\
& B=\frac{2}{n \pi}, \quad C=\alpha+\lambda_{1} n^{2} \pi^{2}, \\
& B_{1}=\frac{A}{C_{1}}, \quad B_{2}=\frac{B-A}{C_{3}}, \quad \beta_{1}=\beta(e-2) \text {, } \\
& A_{1}=\frac{2 \beta\left((-1)^{n}-1\right)}{n \pi}, \quad B_{3}=\frac{A_{1}}{C_{2}}, \\
& B_{4}=\frac{A}{C_{2}}, \quad \sigma_{1}=\sigma(e-2), \\
& A_{2}=-\frac{2 \sigma\left((-1)^{n}-1\right)}{n \pi}, \quad B_{5}=\frac{B-A}{C_{4}} \text {, } \\
& B_{6}=\frac{A_{2}}{C_{2}}, \quad B_{7}=\frac{B}{C-C_{1}}, \quad B_{8}=\frac{B_{2}-B_{1}}{C-C_{1}} \text {, } \\
& { }_{2}=D_{1} n^{2} \pi^{2} \\
& B_{9}=\frac{B_{1}}{C}, \quad B_{10}=\frac{A}{C}, \\
& A_{3}=\frac{p\left(\left(1-(-1)^{2 n}\right)+2\left((-1)^{2 n}-1\right)\right)}{2 n^{2} \pi^{2}}, \\
& A_{4}=\frac{q\left(\left(1-(-1)^{2 n}\right)+2\left((-1)^{2 n}-1\right)\right)}{2 n^{2} \pi^{2}}, \\
& A_{5}=\frac{2 q\left(6\left((-1)^{n}-1\right)+n^{2} \pi^{2}\right)}{n^{3} \pi^{3}}, \\
& C_{1}=\alpha_{2}+\lambda_{2} n^{2} \pi^{2}, \quad C
\end{aligned}
$$

Then, using equations (16) - (23), we obtain $\theta(x, t)=\theta_{0}(x, t)+\in \theta_{1}(x, t)$ $\varphi(x, t)=\varphi_{0}(x, t)+\in \varphi_{1}(x, t)$ $Y(x, t)=Y_{0}(x, t)+\in Y_{1}(x, t)$ $\left.Z(x, t)=Z_{0}(x, t)+\in Z_{1}(x, t)\right)$
The computations were done using computer symbolic algebraic package MAPLE.

\section{RESULTS AND DISCUSSION}

We solve the systems of partial differential equations describing smouldering of a porous carbonaceous rod analytically. We decouple the equations using parameter-expanding method and solve the resulting equations using eigenfunctions expansion technique.

Equations (40) - (47) are the Analytical solutions obtained for equations (10) - (14) and these analytical solutions are computed and depicted graphically using the following parameters values

$$
\begin{aligned}
& \delta=0.4, \lambda_{1}=0.3, \lambda_{2}=0.3, D_{1}=0.3, \in=0.01, \\
& k_{1}=k_{2}=1, \sigma=0.2, \beta=0.2, \alpha_{1}=1, \alpha_{2}=10 .
\end{aligned}
$$

The following figures explain the temperatures and species mass fraction distributions against different dimensionless parameters.

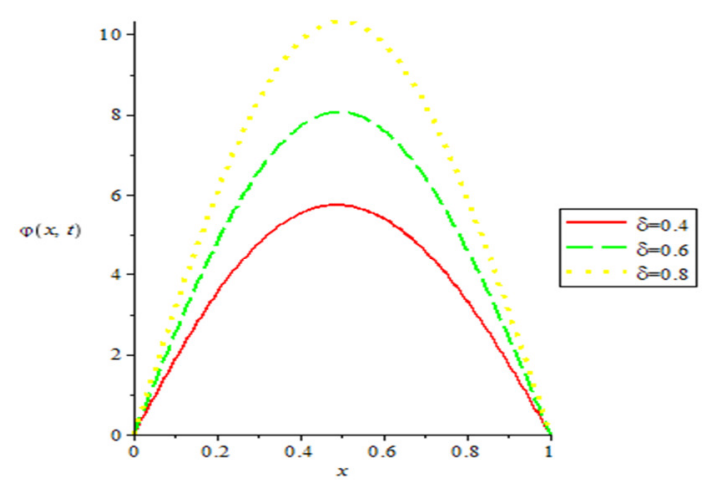

Fig 2: Variation of gas phase temperature $\varphi(x, t)$ with FrankKamenetskii number $\delta$.

number enhances the consumption of Oxygen. This is also as a result of increase in heat of reaction.

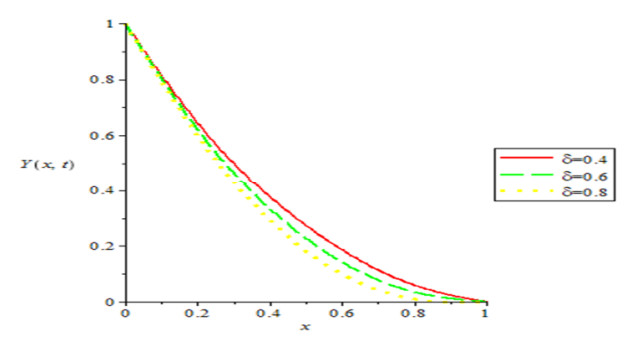

Fig 2 shows the effect of Frank-Kamenetskii number $(\delta)$ on the gas phase temperature profile. It is observed that the maximum gas phase temperature increases. Clearly, the Frank-Kamenetskii number enhances the maximum gas phase temperature. This is as a result of increase in heat of reaction.

Fig 3 depicts the effect of Frank-Kamenetskii number $(\delta)$ on the Oxygen mass fraction profile. It is observed that the Oxygen mass fraction decreases with distance. Clearly, the Frank-Kamenetskii 
Fig 3: Variation of Oxygen mass fraction $Y(x, t)$ with Frank-Kamenetskii number $\delta$.

Fig 4 presents the effect of Frank-Kamenetskii number $(\delta)$ on the gas product mass fraction profile. It is observed that the maximum gas product mass fraction increases. Clearly, the Frank-Kamenetskii number enhances the production gas product. This is as a result of increase in heat of reaction.

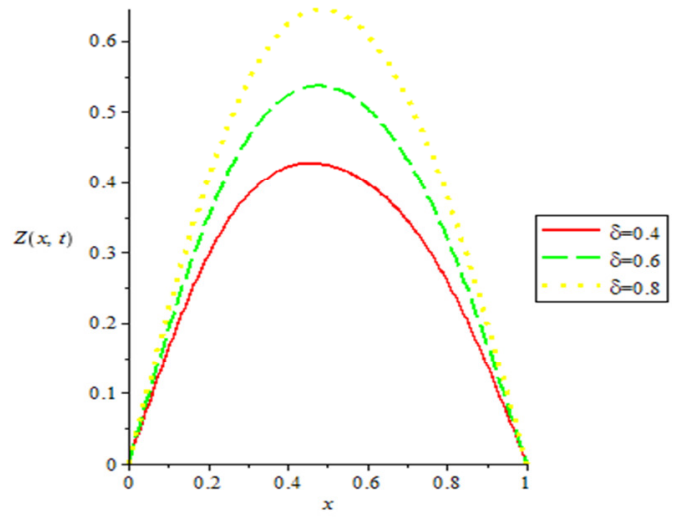

Fig 4: Variation of gas product mass fraction $Z(x, t)$ with Frank-Kamenetskii number $\delta$.

Fig 5 shows the effect of solid phase scaled thermal conductivity $\left(\lambda_{1}\right)$ on the gas phase temperature profile. It is observed that the maximum gas phase temperature decreases. Clearly, the solid phase scaled thermal conductivity decreases the maximum gas phase temperature.

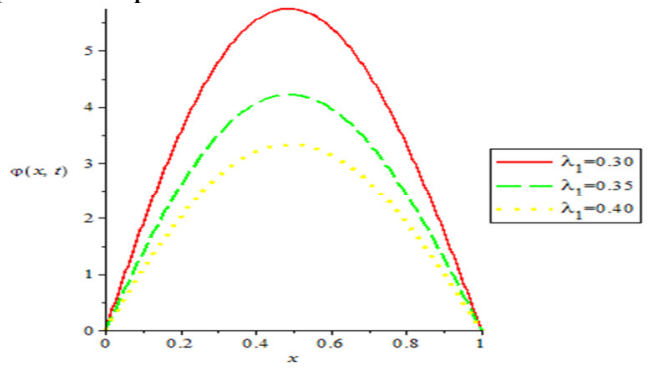

Fig 5: Variation of gas phase temperature $\varphi(x, t)$ with solid phase scaled thermal conductivity $\lambda_{1}$

Fig 6 depicts the effect of solid phase scaled thermal conductivity $\left(\lambda_{1}\right)$ on the Oxygen mass fraction profile. It is observed that the Oxygen mass fraction decreases with distance. Clearly, the solid phase scaled thermal conductivity decreases the consumption of Oxygen.

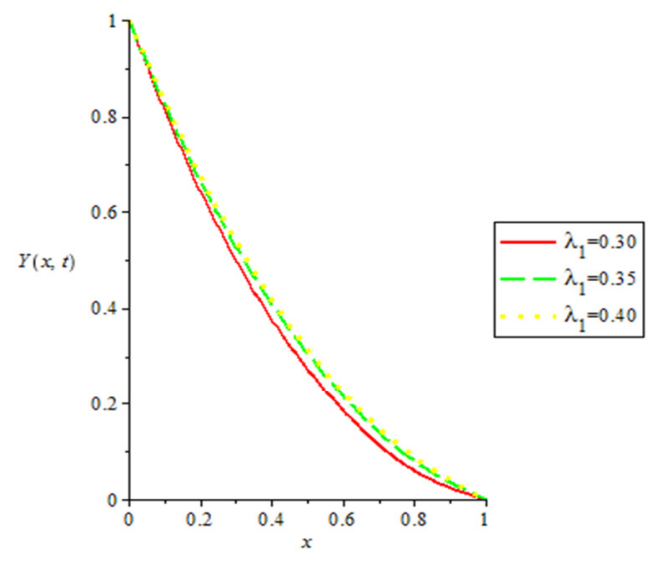

Fig 6: Variation of Oxygen mass fraction $Y(x, t)$ with solid phase scaled thermal conductivity $\lambda_{1}$

Fig 7 presents the effect of solid phase scaled thermal conductivity $\left(\lambda_{1}\right)$ on the gas product mass fraction profile. It is observed that the maximum gas product mass fraction decreases. Clearly, the solid phase scaled thermal conductivity decreases the production of gas product.

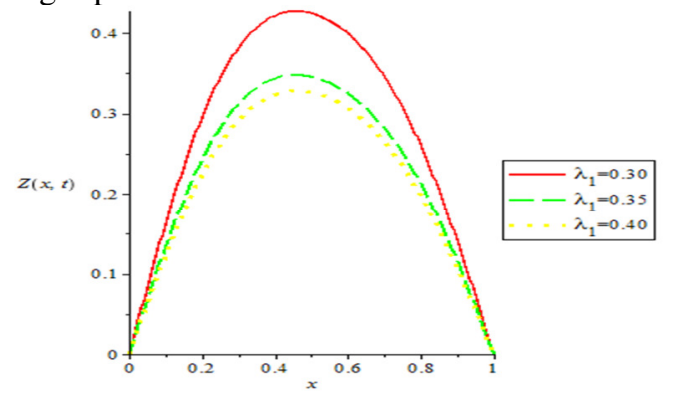

Fig 7: Variation of gas product mass fraction $Z(x, t)$ with solid phase scaled thermal conductivity $\lambda_{1}$

Fig 8 shows the effect of gas phase scaled thermal conductivity $\left(\lambda_{2}\right)$ on the gas phase temperature profile. It is observed that the maximum gas phase temperature increases. Clearly, the gas phase scaled thermal conductivity increases the maximum gas phase temperature. 


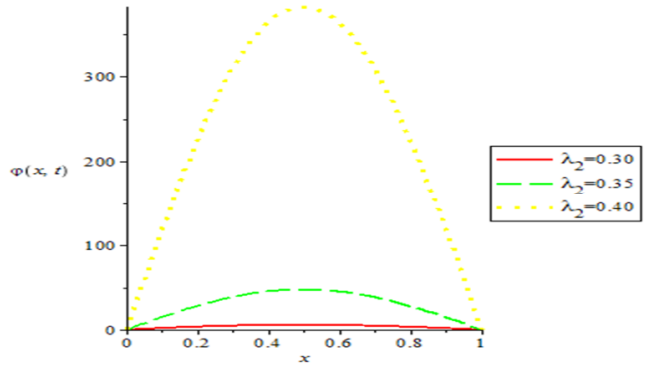

Fig 8: Variation of gas phase temperature $\varphi(x, t)$ with gas phase scaled thermal conductivity $\lambda_{2}$

Fig 9 depicts the effect of gas phase scaled thermal conductivity $\left(\lambda_{2}\right)$ on the Oxygen mass fraction profile. It is observed that the Oxygen mass fraction decreases with distance. Clearly, the gas phase scaled thermal conductivity increases the consumption of Oxygen.

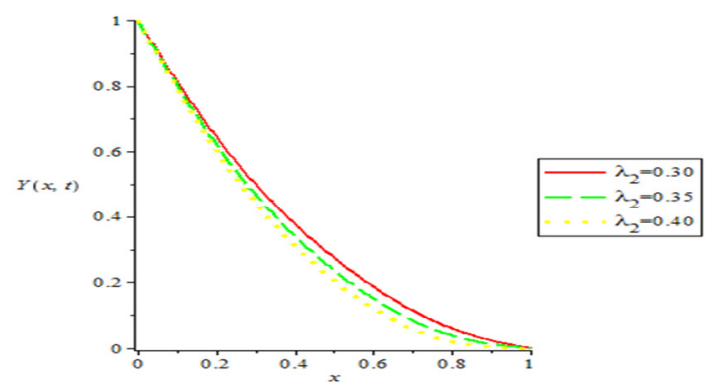

Fig 9: Variation of Oxygen mass fraction $Y(x, t)$ with gas phase scaled thermal conductivity $\lambda_{2}$.

Fig 10 presents the effect of gas phase scaled thermal conductivity $\left(\lambda_{2}\right)$ on the gas product mass fraction profile. It is observed that the maximum gas product mass fraction increases. Clearly, the gas phase scaled thermal conductivity increases the production of gas product.

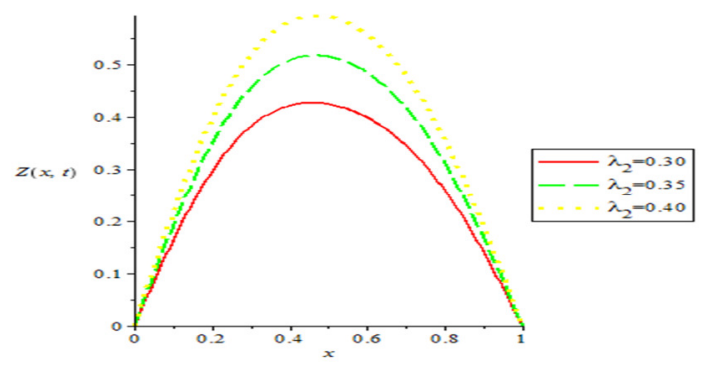

Fig 10: Variation of gas product mass fraction $Z(x, t)$ with gas phase scaled thermal conductivity $\lambda_{2}$
Fig 11 depicts the effect of species diffusion coefficient $\left(D_{1}\right)$ on the gas product mass fraction profile. It is observed that the maximum gas product mass fraction increases. Clearly, the species diffusion coefficient increases the production of gas product.

Note that the effects observed in figs 2 - 11 are of great economic importance, since smoulder waves, which occur naturally, are undesirable, so the goal is to prevent or extinguish them. So there is need to identify the control parameters.

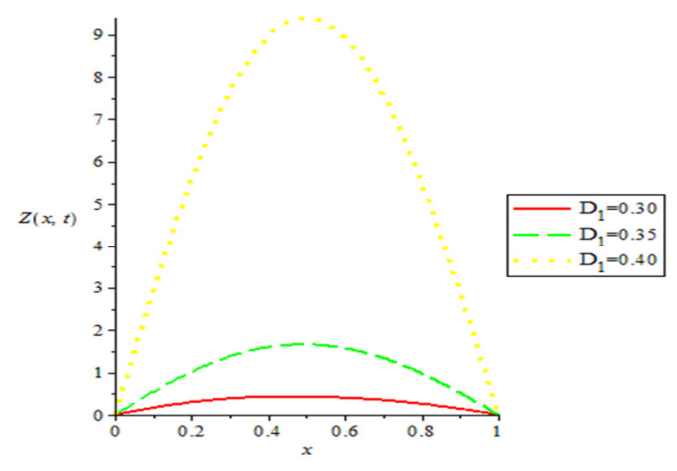

Fig 11: Variation of gas product mass fraction $Z(x, t)$ with species diffusion coefficient $D_{1}$

Conclusion: We have formulated and solved analytically a mathematical model describing smouldering of a porous carbonaceous rod to determine the concentration and temperature distributions. In particular, we have proved by actual solution method that the model formulated has a unique solution for all $t \geq 0$. We decoupled the equations using parameter expanding method and solved the resulting equations using Eigen functions expansion technique. Finally, we have provided the graphical summaries of the system responses.

\section{REFERENCES}

Ayeni, RO (1982). On the explosion of Chainthermal Reactions. J. Austral. Math. Soc. (Series B). 24: 194-202.

Hall, JR (2004). The smoking-material fire problem. Fire Analysis and Research Division of the National Fire Protection Association: Boston, MA.

He, JH (2006). Some asymptotic methods for strongly nonlinear equations, Int. J. Modern Phys. B. 20(10): 1141 - 1199 . 
Kinbara, T; Endo, H; Sega, S (1967). Downward Propagation of Smoldering Combustion through Solid Materials. $11^{\text {th }}$ Symp. Combust., Pittsburgh, Pennsylvania. 525 - 531 .

Myint-U, T; Debnath, L (1987). Partial Differential Equation for Scientists and Engineers. PTR Prentice -Hall, Englewood Cliffs, New Jersey 07632

Ohlemiller, TJ (1985). Modeling of Smoldering Combustion Propagation. Prog. Energy Combust. Sci. 11: 277-310

Olayiwola, RO (2012). Effects of Some Thermophysical Properties on the Propagation of forward and Opposed Smouldering Combustion. The Pacific Journal of Science and Technology. 13(2): 108 - 118. http://www.akamaiuniversity.us/PJST.htm
Olayiwola, RO; Olatunji, SO; Ajao, SO; Waheed, AA; Lanlege, DI (2013). Effect of FrankKamenetskii Parameter on the Propagation of forward and Opposed Smouldering Combustion. Journal of the Nigerian Association of Mathematical Physics. 23: $203-214$.

Rein, G (2009). Smouldering Combustion Phenomena in Science and Technology. International Review of Chemical Engineering, Vol 1:3 - 18. http://www.era.lib.ed.ac.uk/handle/1842/1152

Rostami, A; Murthy, J; Hajaligol, M (2002).

Modeling of a smoldering carbonaceous rod. Fuel Chemistry Division Preprints. 47(1): 205 - 208. 